\title{
Psychiatry amid COVID-19: the Sri Lankan experience
}

\author{
SR Perera, CU Suraweera
}

\section{SL J Psychiatry 2020; 11(1): 5-7}

The COVID-19 pandemic has brought the world to its knees. It has spread to over 200 countries with more than two million infected persons and nearly 200,000 deaths at the time of writing this commentary.

Communicable diseases however are not new and have been in existence from the pre-historic era. As far back as 430 BC, history records a disease with fever, thirst, bleeding and skin lesions that killed as much as twothirds of the world population (1). Since then, the world has survived several pandemics of plague, leprosy, smallpox, cholera and HIV/AIDS. SARS and MERS were more recent epidemics (2).

We have seen health services all over the world struggling to cope with the onslaught of COVID 19. On the sideline, services for vulnerable persons such as the mentally ill are extremely challenged amidst conflicting demands on the health system.

\section{Provision of psychiatric services}

The World Health Organization (WHO) has emphasized the need to ensure availability of essential generic psychotropic medications at all levels of health care, and that people living with long-term mental health conditions will need uninterrupted access to their medication without sudden discontinuation $(3,4)$.

Furthermore, it is also essential to ensure that patients with psychiatric morbidity are not overlooked within nonpsychiatric settings. In the Sri Lankan context, the social and geographical restrictions due to a prolonged curfew to combat spread of COVID 19, has led to mental health services having to adapt and rise to the challenge of continuing psychiatric care in a rapidly evolving environment.

\section{Challenges to continuing quality care}

The human rights law states that in the context of serious public health threats and public emergencies threatening the life of the nation, restrictions on some healthcare rights can be justified. In Sri Lanka, several psychiatry units were the first wards to be taken over as COVID-19 treatment units or quarantine units. Additionally, some psychiatry outreach and community visits were curtailed temporarily. This inadvertently led to the violation of the right to treatment in those with mental disorders.

Maintaining adequate staff to continue services was another challenge. While some team members were enthusiastic and committed to work, others were reluctant due to anxiety and fear of acquiring the infection and also infecting family members. There was also a local need to deploy staff to COVID specific units. This redistribution of experienced mental health personnel to other units is ridden with controversy, as some psychiatrists argue that such a move would almost certainly worsen overall prognosis, and risk deterioration in mental and physical health in patients with mental illness (5).

Sri Lanka has a publicly funded comprehensive network of psychiatric services covering the island run by the Ministry of Health. However, the psychiatric service is largely hospital and outreach clinic based. Community facilities for follow-up of psychiatric patients by general practitioners is almost nonexistent. Patients routinely attend the psychiatry clinics to obtain medication.

To compound the situation, in most instances records have been maintained manually. The pharmacies did not have a transcript of the medication. Therefore, there were obstacles to continuing prescriptions during the curfew period, even though a majority of patients were dependent on the free medications dispensed by hospital pharmacies.

In keeping with the WHO recommendation that an epidemic calls for partnerships that link a hospital to local health care workers, service providers, and the community, mental health workers worked beyond their normal duties and utilized postal services and primary health care staff to deliver medication to patients' homes. Circulars were issued to Medical Officers of Health and general practitioners on how to administer depot medication and monitor blood counts of patients on clozapine. Public messages through the electronic media including online messaging platforms and social media were used to issue public messages on continuing care and accessing psychiatric care. A comprehensive list of hotline numbers was made available so that psychiatry teams could be contacted round the clock. 
Ensuring a steady supply of medication was the next challenge. There were limitations in importation of medicines due to travel restrictions; and issuing of medication in advance for several months at a time lead to a shortage of stocks. Constant liaison was necessary with the drug procurement agencies and the Ministry of Health to ensure a steady supply of essential medicines. Emergency procedures such as electro-convulsive therapy (ECT) were also temporarily curtailed in some instances. ECT requires close exposure to aerosols of patients that may put the anesthetist, the medical officer who administers ECT and the assistant at risk. This risk had to be balanced with the risk of harm by not administering ECT in patients suffering from illnesses such as severe depressive disorder or postpartum psychosis with suicidal ideation.

\section{Facing new challenges}

Certain behaviours shown by patients themselves placed them at increased risk of being infected with COVID 19. The most vulnerable group were those with substance dependence, whose drug seeking behavior in spite of lockdown put themselves and others at risk. The epidemiologists confirmed that certain clusters of infection and spread were among drug users. Therefore many with opioid dependence, including mothers with children, were forcibly sent to quarantine facilities. Quarantine can be an unpleasant experience for those who undergo it. The loss of freedom, separation from family, uncertainty over disease status and boredom can lead to adverse emotions (6).

A national action plan was formulated with the Sri Lanka College of Psychiatrists taking the leadership role, to develop services for patients with mental health needs and at risk of COVID 19. Steps were taken to establish a separate quarantine ward at the National Institute of Mental Health to manage patients with suspected COVID-19, with facilities for isolation.

Island-wide measures were initiated in collaboration with the National Dangerous Drug Control Board (NDDCB) to provide evidence informed interventions for those presenting with substance withdrawal and dependence and those in quarantine centres.

\section{Addressing COVID-19 related anxiety}

Given the emergence of a previously un-encountered and unknown disease like COVID-19, it was not surprising that it caused widespread fear and anxiety among the public. The sensational media reporting on COVID-19 inculcated further worry and helplessness. This led to the formation of harmful stereotypes and increased stigma, that resulted in a dangerous situation where those with symptoms did not seek immediate health care.
Furthermore, there was a risk of this stigma extending to their immediate family, particularly in the event of a death. These were aspects of COVID-19, psychiatrists had to address in the media.

Freedom of movement protects the right of everyone to leave any country, to enter their country of nationality, and the right of everyone in a country to move freely in the whole territory of the country. While lockdown and curfew infringes on this right, the restrictions on movement, physically separated some family members from each other, while in other instances left some family members confined to each other's company for prolonged periods. While for some it was an opportunity to bond and refresh relationships, for others it led to worsening frustration and interpersonal conflicts. There has been concern about the possible increase in interpersonal and domestic violence.

Similar to what was experienced during the outbreaks SARS, we have to anticipate psychological distress, especially within the high-risk groups (7). Psychological first aid may help mitigate the psychological distress (8). Ongoing assessment and appropriate support for emerging psychological issues is a necessity (9).

Healthcare workers on duty round the clock during a pandemic of this nature are likely to suffer from moral injury. This is the psychological distress stemming from actions, or lack of actions, which violate one's moral or ethical code. This may cultivate shame, guilt and disgust. Whether this progresses to post traumatic grief or other significant psychiatric disorders will depend partly on how they are supported before, during and after such challenging incidents.

Evidence suggests that team leaders have a vital role in providing early support and preparation, without false reassurances, in order to reduce the risk of mental health issues. Recommendations are made to engage in Schwartz rounds, which provide an opportunity for staff to reflect on the emotional aspects of their work (10).

The Sri Lanka College of Psychiatrists set up a free helpline manned by a Consultant Psychiatrist round the clock, for frontline workers requiring psychological assistance.

\section{Opportunities for new initiatives}

Initiatives were taken to assist smokers to stop their habit. While focusing on the negative physical and psychosocial effects of tobacco, the effects of smoking on contracting COVID-19 were also highlighted as a negative reinforcer to establish, promote and maintain smoking cessation (11). 
The sale of alcohol was banned by the government during the lockdown and this was a good opportunity for those with alcohol use problems to seek help. Extensive discussions were initiated by government and non-government stakeholders, to develop a comprehensive approach to combat substance dependence.

Telemedicine has arrived to both government and private sectors in Sri Lanka. Although it may not reach all sections of the community, it appears to be a positive development (12). The crises also brought to the forefront the need for electronic patient record keeping in the public health sector.

\section{Conclusions}

The COVID-19 crises provided challenges and opportunities. It more than ever highlighted the leadership role psychiatrists have to play in disaster situations. The ability to adapt to change and develop urgent guidelines and protocols is imperative to meet the needs of our service users. The pandemic no doubt has launched a new and different way of working.

\section{Acknowledgements}

The authors acknowledge Prof. Shehan Williams, Dr. Sajeewana Amarasinghe and Dr. Prabath Wickrama for the valuable guidance in developing this manuscript.

\section{Conflicts of interest}

None declared

$$
\begin{aligned}
& \text { SR Perera, Senior Lecturer in Psychiatry, Faculty of } \\
& \text { Medicine, University of Peradeniya, Sri Lanka } \\
& \text { CU Suraweera, Senior Lecturer in Psychiatry, Faculty } \\
& \text { of Medicine, University of Colombo, Sri Lanka } \\
& \text { Corresponding author: CU Suraweera } \\
& \text { Email: dr.chathurie@gmail.com } \\
& \text { (iD) http://orcid.org/0000-0002-7542-5068 }
\end{aligned}
$$

\section{References}

1. Jarus O. 20 of the worst epidemics and pandemics in history [Internet]. Live Science 2020 [updated $2020 \mathrm{Mar}$ 20; cited 2020 Apr 20]. Available from: https:// www.livescience.com/worst-epidemics-and-pandemicsin-history.html

2. World Health Organization (WHO). Past pandemics [Internet]. WHO 2020 [cited 2020 Apr 20]. Available from: http:/www.euro.who.int/en/health-topics/communicablediseases/influenza/pandemic-influenza/past-pandemics

3. World Health Organization (WHO). Coronavirus disease (COVID-2019) Situation report [Internet]. WHO 2020 [cited 2020 Apr 20]. Available from: https://www.who.int/ emergencies/diseases/novel-coronavirus-2019/situationreports

4. World Health Organization (WHO). Hospital preparedness for epidemics. Geneva: World Health Organization; 2014.

5. Cullen W, Gulati G, Kelly BD. Mental health in the Covid19 pandemic, QJM 2020 Mar 30. pii: hcaa110.

6. Brooks SK, Webster RK, Smith LE, Woodland L, Wessely $\mathrm{S}$, Greenberg N, et. al. The psychological impact of quarantine and how to reduce it: rapid review of the evidence. Lancet 2020; 395(10227): 912-92.

7. Mohammed A, Sheikh TL, Gidado S, Poggensee G, Nguku P, Olayinka A, et al. An evaluation of psychological distress and social support of survivors and contacts of Ebola virus disease infection and their relatives in Lagos, Nigeria: A cross sectional study. BMC Public Health 2015;15: 824.

8. Everly GS Jr, Flynn BW. Principles and practical procedures for acute psychological first aid training for personnel without mental health experience. Int J Emerg Ment Heal 2006; 8(2): 93-100.

9. Sim M. Psychological trauma of Middle East Respiratory Syndrome victims and bereaved families. Epidemiol Heal 2016; 38: e201605.

10. Greenberg N, Docherty M, Gnanapragasam S, Wessely S. Managing mental health challenges faced by healthcare workers during covid-19 pandemic. BMJ 2020; 368: m1211. doi: 10.1136/bmj.m1211.

11. Vardavas CI NK. COVID-19 and smoking: A systematic review of the evidence. Tob Induc Dis 2020; 18(20).

12. Lurie N CB. The Role of Telehealth in the Medical Response to Disasters. JAMA Intern Med 2018; 178(6): 745-6. 